\title{
RETENTION OF PROPRIOCEPTIVE RECALIBRATION FOLLOWING VISUOMOTOR ADAPTATION
}

\author{
NILUFER NOUROUZPOUR
}

\author{
A THESIS SUBMITTED TO THE \\ FACULTY OF GRADUATE STUDIES \\ IN PARTIAL FULFILLMENT OF THE \\ REQUIREMENTS FOR THE DEGREE OF \\ MASTER OF SCIENCE
}

GRADUATE PROGRAM IN KINESIOLOGY AND HEALTH SCIENCE

YORK UNIVERSITY

TORONTO, ONTARIO

APRIL 2014

(C) NILUFER NOUROUZPOUR, 2014 


\section{Abstract}

This thesis builds on our laboratory's recent findings that visuomotor adaptation following reaches with a misaligned cursor not only induces changes in an individual's motor output, but their proprioceptive sense of hand position as well. Long-term changes are seen in motor adaptation, however very little is known about the retention of changes in felt hand position. We sought to evaluate whether this recalibration in proprioception, following visuomotor adaptation, is sufficiently robust to be retained the following day ( 24 hours later), and if so, to determine its extent. Visuomotor adaptation was induced by having subjects perform reaches to visual targets using a cursor representing their unseen hand, which had been gradually rotated $45^{\circ}$ counterclockwise. Motor adaptation and proprioceptive recalibration were determined by assessing subjects' reach aftereffects and changes in hand bias, respectively. We found that subjects adapted their reaches and recalibrated their sense of hand position following training with a misaligned cursor, as shown in Cressman and Henriques (2009). More importantly, subjects who showed proprioceptive recalibration in the direction of motor adaptation on Day 1 did retain changes in felt hand position and motor adaptation on Day 2. These findings suggest that in addition to motor changes, individuals are capable of retaining sensory changes in proprioception up to 24 hours later. 


\section{Acknowledgements}

I believe in lifelong learning and growth, "to find out what's inside you, to make your soul grow"; I'd like to express my deepest gratitude to my supervisor Dr. Denise Henriques for the opportunity to do just that. Thank you Denise! I will always appreciate your guidance, teachings, motivation, as well as your availability to your students. Thank you for the wonderful years I was a member of your lab.

My sincerest thanks go to my co-supervisor Dr. Erin Cressman, I am grateful for all your feedback, guidance, and positivity.

I would also like to say thank you to my thesis committee members Dr. W. Dale Stevens, for his time and insightful comments, as well as Dr. Lauren Sergio - your passion for your work is inspiring, thank you for all your help.

Thank you my colleagues Simona Monaco, Holly Clayton, Ahmed Mostafa, Victoria Barkley, Maria Ayala, Steven Jesin, Roozbeh Kamran-Disfani, as well as our programmer Errol Cheong for their feedback and companionship; I wish you all great success and happiness in your future endeavors. To my good friends David Albines and Mark Davids, you've made my experience especially enjoyable and memorable, thank you for your support and the excessive amounts of coffee.

None of this would have been possible without the support of my family. A big thank you to my father Manoucher, my mother Saadet, and my sister Sahar, as well as my best friend Kyle; your love and encouragement know no bounds. 


\section{TABLE OF CONTENTS}

TITLE PAGE

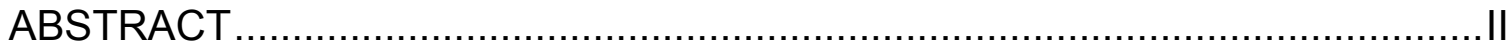

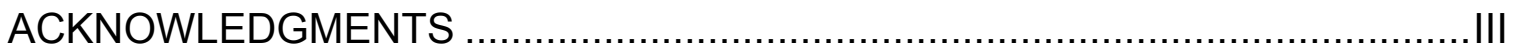

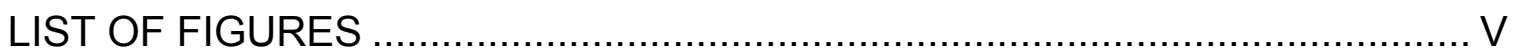

INTRODUCTION

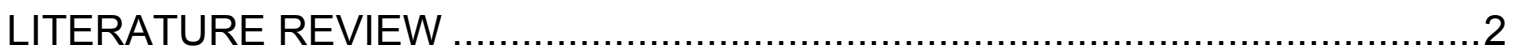

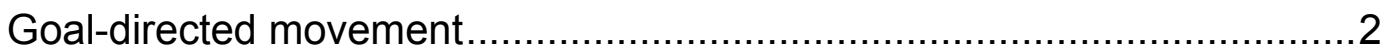

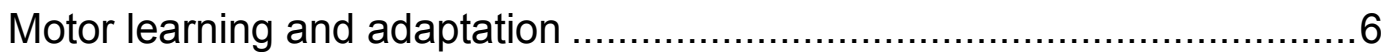

Proprioceptive recalibration following visuomotor adaptation ..................10

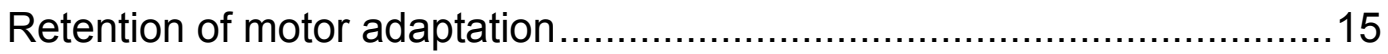

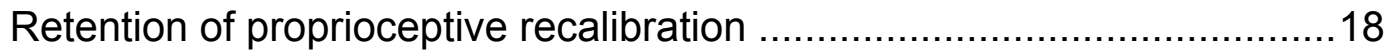

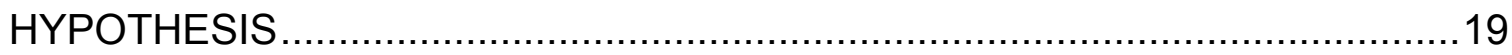

METHODS

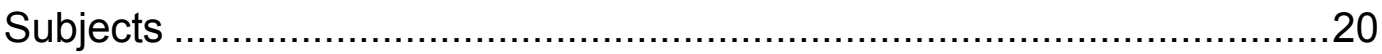

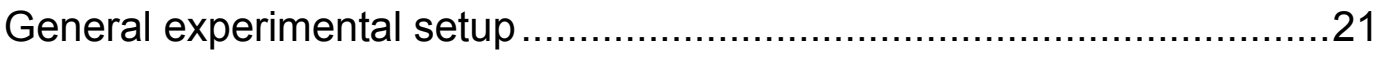

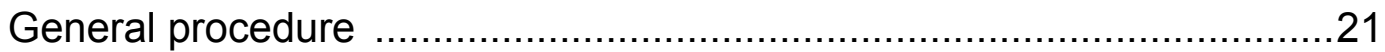

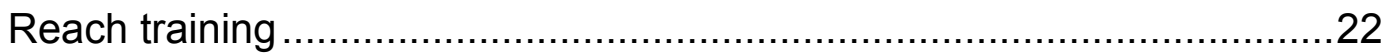

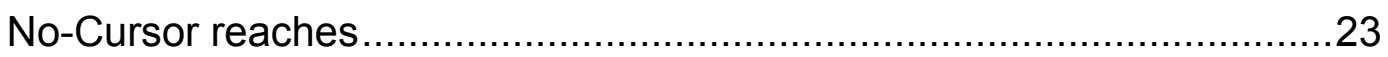

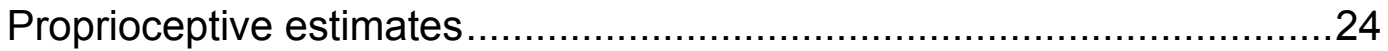

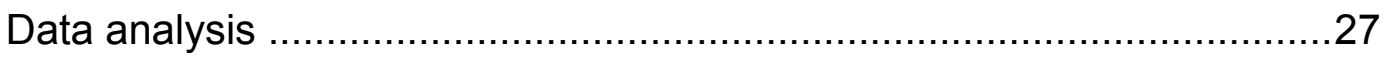

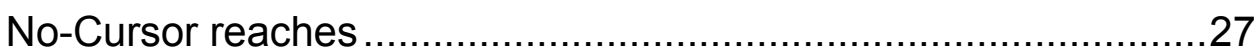

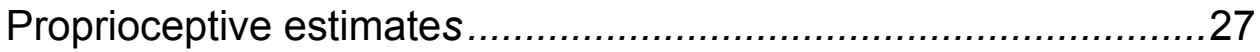

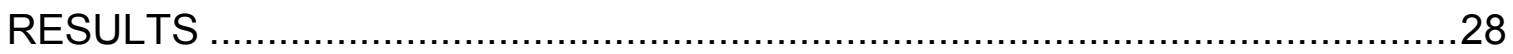

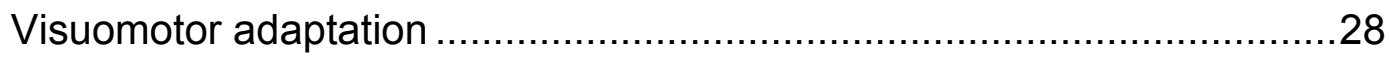

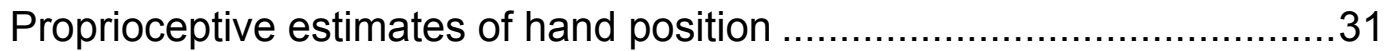

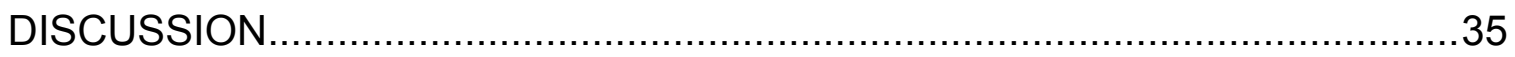

Proprioceptive and motor changes following visuomotor adaptation ........36

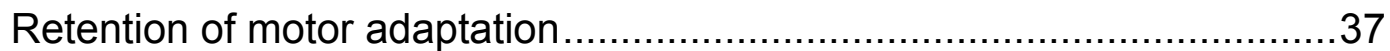

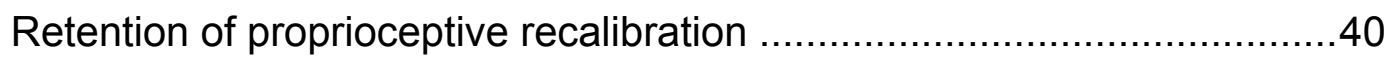

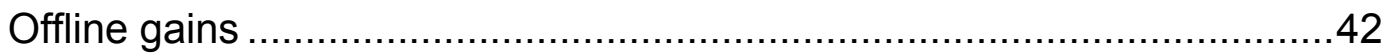

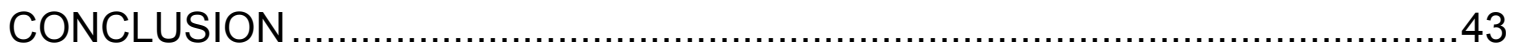

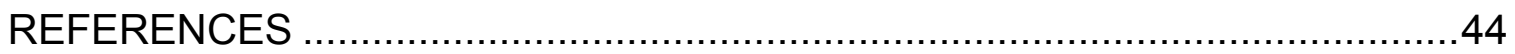

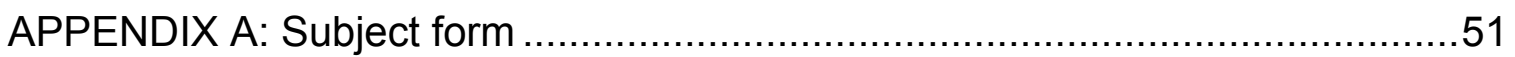




\section{LIST OF FIGURES}

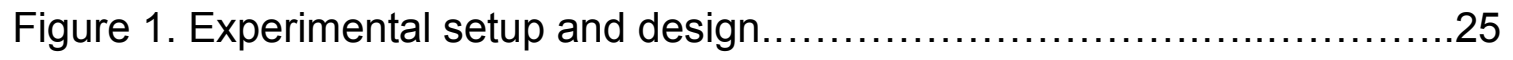

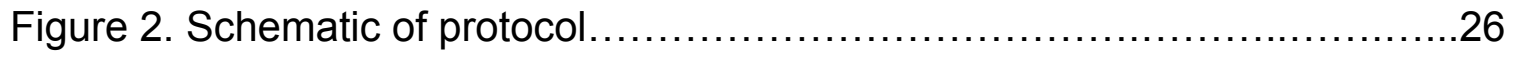

Figure 3. Reach aftereffects.....................................................

Figure 4. Proprioceptive recalibration (multipanel) .................................34 


\section{Introduction}

The ease with which one can accurately reach to a doorbell can lead one to misconceive the action as simple, whereas in reality it involves an array of complex underlying neural transformations. In order to place the limb at a desired spatial location, goal-directed movements require the central nervous system to interpret sensory information (e.g. vision, proprioception) regarding the target and hand's position, thereafter transforming this information into the appropriate motor commands (Jeannerod 1988). In most cases this sensory information is congruent, such that the position one sees and feels their hand overlap. However, under circumstances where this is not the case (e.g. when reaching towards an object under the water's surface), the brain will respond by learning a new visuomotor mapping, thus modifying the hand's subsequent motor commands accordingly. Specifically, when presented with altered visual feedback of their hand, such as while wearing prism goggles or in virtual reality environments, subjects compensate by adjusting their motor commands in order to maintain movement accuracy, thereby gradually reducing the error signal experienced when desired and actual movement outcome are distinct; this process is referred to as visuomotor adaptation. When visual feedback is removed whilst performing open-loop reaches, individuals will continue to make adapted movements and these reach errors, termed aftereffects, provide a measure of motor learning. For instance, following training with a cursor that is rotated relative to their hand in a virtual reality environment, subjects' open-loop 
movement trajectories will remain deviated in the direction of adaptation. These aftereffects, indicating changes in the motor system, can last up to a year following training (Yamamoto et al. 2006).

In addition to motor changes, visuomotor adaptation has been shown to result in short-term sensory changes. Previous studies examining reaching with altered visual feedback of the hand have suggested that visuomotor adaptation is partially due to the recalibration of proprioception (felt joint or limb position in space) to match its visual representation (Hay et al. 1965; Redding and Wallace 2004; Bernier et al. 2005; Redding et al. 2005; Simani et al. 2007). At present, the persistence of these proprioceptive changes following visuomotor adaptation has been left unexplored. Our study aims to investigate whether proprioceptive recalibration and motor adaptation are retained after a 24-hour period following training, and if found, the magnitude of this effect. These results will provide insight into the effects of a longer time-lapse on the retention of proprioceptive recalibration following visuomotor adaptation as well as the relationship between the process of motor adaptation and proprioceptive recalibration.

\section{Literature review}

\section{Goal-directed movement}

Our daily lives incorporate a multitude of interactions with our environment and a significant portion of these actions is comprised of purposeful, goaldirected, movements. For the average human being, reaching for our phones or 
picking up a cup of coffee are seemingly simple tasks to perform, yet they are governed by complex processes integrated by our brain and body. Once the central nervous system (CNS) selects a target or goal for the hand to reach, a motor plan is computed and motor commands needed to achieve the goal are generated.

Vision and proprioception (mentioned below) provide sensory information critical for the estimation of limb position and thus, successful regulation of goaldirected movements. The way this sensory input is processed depends on the demands of the motor task at hand; van Beers and colleagues (1999) have shown that motor performance is improved when one has access to information from multiple sensory modalities regarding limb position, when compared to information from only one source. Depending on the stage of movement planning (Sober and Sabes 2003, 2005), target position (van Beers et al. 1996, 1998, 1999), and target modality (Sober and Sabes 2005), the brain can select different combinations of sensory input (i.e. vision, proprioception) to localize one's hand. For instance, movement vector (or pathway) planning relies mostly on visual signals, whereas the computation of the intrinsic motor command (or muscle signals) needed to correctly move the hand relies heavily on proprioception (Sober and Sabes 2005).

Studies have found that when visual information was present, subjects could reach (Prablanc et al. 1979) and point (Admiraal et al. 2003) to targets more accurately, as well as produce more accurate movements (Woodsworth 1899) compared to movements completed in the absence of vision. Although it 
has been suggested that only brief visual samples are generally sufficient for reasonably precise closed-loop control (Elliott et al. 1995), vision still remains of significant importance for optimal movement control even after considerable practice (Proteau 1995). This is especially true in the final, homing-in, phase compared to the initial ballistic phase in rapid-aiming movements, so that the hand can precisely reach its target (Woodsworth 1899).

The location of the hand can also be estimated using proprioception. Proprioceptors are sensory receptors that convey information to the CNS about the body's position or movement in space and can be found primarily in muscles, tendons, and joints. Studies involving individuals lacking proprioceptive input have found deficits in most aspects of their motor ability and suggest that proprioception is essential in achieving fine controlled movements in the absence of vision (Rothwell et al. 1982). Muscle spindles are a type of proprioceptor which provide afferent signals about changes in length (including velocity, acceleration, and position) of the muscle in which they are located. They are found in higher numbers in muscles that are necessary for fine movements than those used for posture or gross motor skills. Information from muscle spindles regarding the kinematic properties of muscles that cross joints also help the CNS sense the relative position of body segments (Burgess and Clark 1969). They are found parallel to extrafusal muscle fibers (controlled by alpha motor neurons), thus any changes in length in the latter will also stretch intrafusal muscle fibers and its respective sensory endings, both found within the muscle spindle, increasing the sensory endings' firing rate. Tendon vibrations have been used in humans to 
investigate kinesthetic illusions, causing a deceptive sense of movement and altered joint position (Goodwin et al. 1972). The Golgi tendon organs are encapsulated sensory receptors, found in the transitional area between muscle fibers and tendons, which are sensitive to changes in muscle tension. A tendon organ is innervated by a single group $1 \mathrm{~b}$ axon that enters its capsule, demyelinates, and branches around collagen strands. Golgi tendon organs are found in-series in the aforementioned junction and stretching the tendon organ (and thus, collagen fibers) will compress the nerve endings of the axon, causing it to fire and signal the tension developed by the muscle. In addition to other anatomical sensors in the body, muscle spindles and Golgi tendon organs convey critical sensory signals used by the CNS to accurately deduce the body's felt position or movement in space.

Movement can be broken down into two main components: kinematics and dynamics. The geometry and speed of a movement are termed the kinematics, whereas the forces required to produce the movement are the dynamics (Krakauer 2006). In order to produce a successful goal-directed movement, the CNS must estimate the kinematic and dynamic changes required to reach the intended target, while visual and proprioceptive signals convey the current location of the hand. Internal models are theorized to be neural processes which allow us to perform goal-directed actions both prior to and after movement onset (Wolpert and Kawato 1998). During computation of inverse kinematics, the end effector position is defined and the state vector of the structure, which would produce the desired trajectory or final position, must be 
derived (Kawato 1999). In other words, with the knowledge of your current hand position, the motor system must determine the necessary joint angles to move your hand to a specific target. In addition, the motor system also needs to compute the amount of torque that must be produced at each joint for the hand to reach the target; this is termed inverse dynamics. In forward kinematics, the motion of the joints are explicitly specified, which will compute the end effector position; an efference copy is issued from the motor command, stemming from sensory signals detecting length changes in the muscle, and will predict the resulting sensory consequences. The computed estimation of your hand's motion as a result of specific muscle forces is termed forward dynamics (Wise and Shadmehr 2002).

\section{Motor learning and adaptation}

In our everyday lives we effortlessly use a variety of motor skills that have been acquired through practice and interaction with our environment. Skill acquisition involves the learning a novel behaviour, resulting in the expansion of our motor repertoire. Motor adaptation allows us to adapt our well-learned motor skills to new circumstances or environments. For instance, when first learning how to use a keyboard, the co-articulation of a child's finger movements may be very slow and imprecise. However with sufficient practice the child can successfully acquire this motor skill and improvements, such as faster typing speeds while pressing the correct keys, will be apparent. If the child is given a new keyboard with the letter keys in different locations, although the task still 
requires the same skill (i.e. typing), the context will have changed and will result in a decline in performance (i.e. accuracy, speed). By modifying her motor commands, thus reducing the error signal produced when desired and actual movement outcome are distinct, she can compensate for the altered environment. In other words, motor adaptation allows one to return to their original proficiency in a skill, rather than learn a new one.

As previously mentioned, in order to place the limb at a desired spatial location, goal-directed movements require the central nervous system to interpret sensory information regarding the target and hand's position, thereafter transforming this information into the appropriate motor commands (Jeannerod 1988). In most cases this sensory information is congruent, however under circumstances where sensory information is incongruent, the brain will respond by modifying the hand's subsequent motor commands accordingly. To study our control system's capacity to compensate for changes in the sensory and motor relationship, such as growth, injury, and novel environments, motor adaptation can be induced through two main types of perturbations in a laboratory setting. The first type of perturbation involves altering visual feedback, inducing visuomotor adaptation, which can be achieved using prism goggles or virtual reality environments. The second type of perturbation involves adding mechanical disturbances to movement, such as introducing a force-field (Shadmehr and Mussa-Ivaldi 1994) or changing the inertial properties of the moving limb (Bock 1990). A measure of motor adaptation following training with a perturbation is the aftereffect; the continuation of adapted movements following 
the removal of a perturbation. For instance, after completing a reach training task with a cursor that has been gradually misaligned or rotated, removal of visual feedback of the unseen hand's position results in continued deviated trajectories in the direction of adaptation during open-loop (ballistic) reaches. Their presence suggests that there had been formation of an updated or new internal model by the CNS following visuomotor adaptation (Shadmehr and Mussa-Ivaldi 1994; Wolpert et al. 1995; Shadmehr and Moussavi 2000).

Numerous studies have used prism goggles to study visuomotor adaptation in both humans and monkeys. Inverse prisms optically rotate a subject's perceived world around the line of sight by $180^{\circ}$, whereas wedge prisms only displace vision by approximately $5^{\circ}$ to $25^{\circ}$. Shifting the subject's visual field will cause a visual-kinesthetic conflict in the perception of location and subjects initially show signs of severely disrupted visually-guided movements. However, studies have consistently found that with repeated practice subjects can show error reductions in visually-guided behaviour (Harris 1963; 1965; Hay and Pick 1966; Redding et al. 2005; Redding and Wallace 1978; 1988; 1996; 1997; 2001; 2002; 2003; 2006) and in eye movements (Gonshor and Jones 1976). Once the prisms are removed, the strength of the adaptation can be measured by deviations in subjects' movement aftereffects, in the direction opposite to the visual shift.

Visuomotor adaptation can also be induced through the manipulation of a hand-cursor. This perturbation allows for the misalignment of real-time visual feedback of one's hand in a virtual reality environment, without shifting the entire 
visual field, including the target (as done with prisms). Altered visual feedback of the hand can be introduced by manipulating the cursor in a number of ways, such as introducing a rotation, translation, or a novel scaling factor of the visual space. For instance, the cursor can be rotated $30^{\circ}$ clockwise $(\mathrm{CW})$ relative to actual hand motion during reaching movements. In this scenario, subjects gradually adjust their hand movements in a counter-clockwise (CCW) fashion so that their cursor attains the target, even if their actual unseen hand is misaligned from this desired endpoint (Krakauer et al. 1999). Reaching baseline levels of performance only requires approximately 20 trials per target (Krakauer et al. 2000). Similar to prism adaptation, subjects will exhibit aftereffects once the distortion is removed, such that they continue to reach in the direction of adaptation, ascertaining the formation of a new visuomotor mapping by the CNS. Visuomotor adaptation to a rotated cursor has also been shown to transfer between limbs. In particular, Sainburg and Wang (2002) found that opposite arm training improved the initial direction of right arm movements during misaligned training, whereas opposite arm training improved the final position accuracy of left arm movements. Dionne and Henriques (2008) found transfer of adaptation when the image of a subject's hand was mirror-reversed, however transfer did not occur when feedback was a rotated view of their hand.

The exact angle of a rotation has been found to influence adaptation, such that task difficulty increases along with the angle of rotation (Cunningham 1989), whereas adaptation to a previous rotation (Abeele and Bock 2001) or adapting in smaller steps (Buch et al. 2003) can facilitate adapting to larger rotations. In 
addition, aftereffects were found to be larger (Contreras-Vidal and Stelmach 1997) and lasted longer (Klassen et al. 2005) when the distortion had been introduced gradually as opposed to abruptly. When small reach errors arise while training with gradually rotated distortions, it is thought that implicit learning processes are involved; these processes update the internal model and attempt to increase movement accuracy on subsequent trials. When distortions are introduced abruptly, large initial errors are made and subjects employ explicit strategies in their compensatory movements (Redding and Wallace 1996). Implicit learning can also be part of this initial process or occur later on. In a study by Mazzoni and Krakauer (2006), subjects had been instructed on explicit strategies during visuomotor adaptation tasks to counteract the visuomotor distortion; they found that subjects initially showed a decrease in target errors, however, as training proceeded their performance began to deteriorate such that they made increasingly large errors while reaching to the target. This suggests that during visuomotor adaptation, implicit, thus unconscious, motor adaptation will occur regardless of the presence of explicit strategies.

\section{Proprioceptive recalibration following visuomotor adaptation}

In addition to motor changes, visuomotor adaptation has been shown to result in short-term sensory changes, such as proprioceptive recalibration. Changes to felt hand position have been studied using various perturbations, including prism adaptation, virtual reality environments, and force-field adaptation. 
Previous studies examining reaching with altered feedback of the hand have suggested that visuomotor adaptation is partially due to the recalibration of proprioception to match its visual representation (Hay et al. 1965; Redding and Wallace 2004; Bernier et al. 2005; Redding et al. 2005; Simani et al. 2007). In these previous studies, subjects used their adapted hand to perform proprioceptive estimation tasks, thus making it difficult to discern whether recalibration to such targets arose due to motor adaptation. However, changes in felt hand position have been observed using paradigms which do not require subjects to make goal-directed movements, such as the use of two-alternative, forced-choice psychophysical tests, hence eliminating the possibility of a motor confound (Cressman and Henriques 2009; Ostry et al. 2010). For instance, Ostry and colleagues (2010) measured change in felt limb position by having subjects report the direction their hand had been deflected (left or right) during their reaches after training in a force-field paradigm. Cressman and Henriques (2009) had subjects use a robot manipulandum which guided their hand along a constrained pathway to a designated location. Once it attained this final position, a visual or body midline (a non-visual reference point) reference marker appeared and subjects made a forced-choice judgment of the position of their hand relative to the marker (left or right). When tested following visuomotor adaptation, subjects displayed a significant proprioceptive recalibration in the expected direction of motor adaptation. Results from our lab have shown that this recalibration of hand position by approximately $6^{\circ}$, or $20 \%$ of the $30^{\circ}$ distortion, and occurs under a multitude of contexts. 
Cressman and Henriques (2009) had subjects perform the aforementioned proprioceptive estimation task while moving subjects' hands passively (guided by the robot) and actively, following training with either a rotated or translated cursor. They found that following visuomotor adaptation, subjects recalibrated their felt hand position approximately $20 \%$ of the magnitude of the distortion, regardless of cursor manipulation, including when the hand was moved passively or actively during felt hand position estimation tasks. Proprioceptive recalibration following visuomotor adaptation was seen to a similar extent in older adults (mean age $=66.3$ years) (approximately $20 \%$ of the distortion) as young adults (mean age $=27.3$ years). However, older adults had less precision (or higher uncertainty) when having to estimate hand position (Cressman et al. 2010). Clayton et al. (2013) found similar results for individuals with Ehlers-Danlos syndrome, a group of connective tissue disorders characterized by joint hypermobility.

This shift in sense of hand position also arises following adaptation in both the left and right hands of right-handed individuals (Salomonczyk et al. 2012; Mostafa et al. 2014). Transfer of adaptation is thought to have occurred when initial errors in response to the same perturbation are smaller or when the learning rate is faster for the untrained hand following training of the opposite hand. Moreover, transfer can also be measured by comparing changes in aftereffects and hand biases between trained and untrained hands, as seen in a study by Mostafa and colleagues (2014). They sought to investigate whether induced changes in felt hand position could transfer from the trained hand (right 
or left) to the untrained hand (left or right) following visuomotor adaptation. They found intermanual motor transfer from the right trained (dominant) hand to the left untrained (non-dominant) hand, however did not find transfer from the left trained hand to the right untrained hand. In addition, although proprioceptive recalibration had been observed in both trained hands, intermanual transfer of shifts in felt hand position did not occur.

The ability to apply our learned skills in a new context is termed generalization; if it is beneficial it is termed transfer, however if it is unfavorable it is called interference (Krakauer et al. 2006). Generalization patterns have been explored by Cressman and Henriques (2010a) wherein subjects' reaching movements and changes in felt hand position to novel locations were studied following visuomotor adaptation. They found that generalization of reach adaptation was local, in that adaptation was only observed at locations that were close to the trained target direction. Moreover, these aftereffects diminished drastically for targets located $20^{\circ}$ from the trained target, confirming previous studies' observations that generalization patterns in motor adaptation are local (Krakauer 2000; Wang and Sainburg 2005). Interestingly, proprioceptive recalibration generalized quite broadly in comparison, such that changes in felt hand position in the trained location were found to a similar extent for locations up to $90^{\circ} \mathrm{CCW}$ from the trained target direction. In the same study, they also found that after training with small cursor-gains, subjects' reaches had been adapted, whereas felt hand position was not recalibrated. 
In a unique paradigm, Cressman and Henriques (2010b) examined if exposure to solely a sensory discrepancy was sufficient enough to induce reach adaptation and proprioceptive recalibration. Subjects held a robot manipulandum, which guided their hand either actively or passively along a fixed linear path. On each trial, subjects saw the cursor reach the target, however, the fixed path was gradually being rotated $30^{\circ} \mathrm{CCW}$ away from the straight path of the cursor. Misaligning the hand's path (proprioceptive signals) from the cursor's path (visual signals) created a cross-sensory error signal, without a goal-directed movement or, essentially, a sensorimotor error signal. They found that subjects had in fact adapted their reaches by $6^{\circ}$ without the presence of a movement related error signal. They also found that felt hand position in subjects had been recalibrated to a similar magnitude as their reaches were adapted. In a similar experiment by Salomonczyk et al. (2013), they tested subjects' exposure to a $30^{\circ}, 50^{\circ}, 70^{\circ}$ rotated hand-cursor distortion. No additional increases in recalibration or reach adaptation were observed, such that the changes for the $50^{\circ}$ and $70^{\circ}$ distortions were similar in magnitude as changes for the $30^{\circ}$ distortion. Given the similar extent of reach and proprioceptive changes, they suggested that the reach adaptation observed had partially occurred due to changes in subjects' proprioceptive bias. Since $6^{\circ}$ is only $1 / 3$ of typically observed aftereffects in our lab (Cressman and Henriques 2009), it is likely that the usual, larger, aftereffects produced after visuomotor adaptation are associated with the sensorimotor error signal. 
Unlike the cross-sensory error signal, the magnitude of the sensorimotor discrepancy, and thus the distortion, has been found to affect subjects' reaches as well as their shift in felt hand position following visuomotor adaptation. Salomonczyk et al. (2011) had subjects train to reach with a cursor that had been rotated by $30^{\circ}, 50^{\circ}$, and $70^{\circ}$. Although reaches and estimates of felt hand position were directly related to magnitude, they showed the same percent change found in previous work: $20 \%$ of the distortion for proprioceptive recalibration and $50 \%$ for motor adaptation.

\section{Retention of motor adaptation}

Various studies have demonstrated that induced motor changes accompanying visuomotor adaptation remain long after training has ended, and can last from several days up to a year following training. Specifically, after adapting to a perturbation, subjects continue to show reach aftereffects (Yamamoto et al. 2006) or faster relearning when exposed to the same perturbation on a later day (i.e. presence of savings) (Krakauer et al. 1999; Bock et al. 2001; Tong et al. 2002; Caithness et al. 2004; Klassen et al. 2005; Krakauer et al. 2005). For instance, when Klassen et al. (2005) retested subjects one day after they had modified their cursor reaches to an abrupt $30^{\circ}$ rotation, the initial errors produced at the start of the second day were similar to those at the end of training on the first day, suggesting almost complete retention of motor adaptation. Likewise, Caithness et al. (2004) found that when subjects were retested on a $30^{\circ}$ visuomotor rotation 48 hours after initial adaptation, and initial 
deviations in reaching were reduced by approximately half when compared to those produced in the very first block two days before, once again suggesting substantial retention of the visuomotor adaptation.

Bock and colleagues (2001) also demonstrated retention of motor adaptation in an experiment where a sensorimotor discordance was introduced by reversing cursor movement either left-right or up-down. In this study, subjects tracked a moving visual target by shifting the perturbed cursor with a joystick. They were tested again in a second session on the perturbed tracking task at one of various time points ( $8 \mathrm{~min}, 25 \mathrm{~min}, 1 \mathrm{hr}, 2 \mathrm{hr}$, or 1 month later). Their overall performance (the root mean square error of the distance between the cursor and target) was greatly improved; specifically, when retested up to one month later, subjects showed almost complete retention of the adaptation such that tracking errors were observed to be close to end-of-training levels (baseline). Yamamoto et al. (2006) found that in addition to the commonly observed shortterm changes accompanying the process of motor learning, motor memory can be retained for extended periods of time. In this study subjects learned to use a joystick to move a cursor, which was gradually rotated $40^{\circ}$, onto a target. Following training, when reaching in the absence of a cursor, subjects showed compensation in their movement aftereffects for 63 to $91 \%$ of the rotation. More importantly, 59 to $91 \%$ of the compensation was still present one year later, relative to the initial post-test (41-77\% of the visuomotor distortion).

Long-lasting effects on motor performance have also been found following adaptation to other perturbations, such as a velocity dependent force-fields 
(Brashers-Krug et al. 1996; Shadmehr and Brashers-Krug 1997). Shadmehr and Brashers-Krug (1997) found that after subjects had adapted their reaches with a velocity dependent force-field, the level of performance achieved on that first day persisted up to 24 hours later whilst reaching in the same force field. Interestingly, this improvement remained constant when retested 5 months later, suggesting the formation of long-term motor memories following this type of learning.

Retention of motor adaptation is also observed following prism adaptation. In a study by Kitazawa and Yin (2001) found that monkeys could adapt goaldirected reaches while wearing laterally-displacing wedge prism goggles that shifted their entire field of vision. The task required the monkey to reach to a square target zone that would appear on a touch screen, while the view of its hand and the target were blocked by liquid-crystal shutters in front of the eyes at the onset of movement. The monkey was given terminal feedback of its hand's final position and the target once it had touched the screen. Aftereffects more than half the size of the prism displacement were observed after only 50 trials. In addition, after they had increased the number of training trials to 500 , the monkey had shown a similar magnitude of aftereffects up to 48 and 72 hours later. Moreover, humans who had spent 6 weeks adapting their throwing movements while wearing displacing prisms showed almost perfect retention when retested 9,18 , and 27 months following initial training, such that they accurately threw balls near the center of the target, on their first throw, right after donning prism 
goggles. It required the same amount of time for participants to unlearn the adaptation as it did to initially learn it (Martin et al. 1996).

Retention of proprioceptive recalibration

Still unknown is whether changes in hand proprioception persist beyond training, following visuomotor adaptation, the way reach adaptation does. Retention of the change in felt hand position was investigated using a velocitydependent force-field perturbation, and has been shown to persist at least 24 hours after adaptation (Ostry et al. 2010). This change in felt limb position was measured by having subjects report the direction their hand had been deflected (left or right) during their reaches; this was measured at various intervals throughout the experiment, including before, after, and 24 hours following training. The authors observed a perceptual shift of approximately $11 \%$ (compared to peak deviations in aftereffects) of the estimated magnitude of learning following training and interestingly, these sensory changes were also observed to a similar extent 24 hours later. The latter finding suggests that persistent changes in the sensory system are present following adaptation to a velocity dependant force-field. How these somatosensory changes compare with motor changes, such as aftereffects or savings is not known, since changes in movement were not re-measured the following day. In addition, although retention of changes in felt hand position following force-field training is known, its retention following visuomotor adaptation (visual-proprioceptive discrepancy) has not yet been investigated. 


\section{Hypothesis}

Our aim was to test for motor adaptation and proprioception recalibration following visuomotor adaptation and to determine if these changes could be retained following a period of 24 hours. In addition, if these changes were found, we sought to measure the extent of retention for both adaptation and recalibration. We do expect to see a moderate decay in proprioceptive recalibration since normal vision of the hand in the intervening 24 hours would presumably lead to a return of the habitual visual-proprioceptive alignment. If these changes are retained, then we can suggest that sensory memory may play a role in the long-term changes experienced following visuomotor adaptation 


\section{Methods}

\section{Subjects}

60 right-handed participants were recruited from York University (mean age $=23.5 \mathrm{yr}, \mathrm{SD}=5.5 \mathrm{yr}, 43$ women), 39 of which were enrolled in a first-year university psychology course and participated for course credit. All subjects were free from neurological impairments as well as pre-screened for self-reported right-handedness and corrected vision. Data from 14 of the initial 39 subjects were excluded from analyses due to incorrect performance of the Proprioceptive Estimates task (see below). Failure to perform the Proprioceptive Estimates task has been observed on a smaller scale in our previous studies and the large number of participants who needed to be discarded in the present study most likely reflects the brevity of instructions given to our first set of participants. This was later remedied leading to $100 \%$ keep rate for the last 21 subjects. One of the 14 subjects (mentioned above) had been removed because the changes in their proprioceptive bias, although in the expected direction of motor adaptation, fell outside 2 SD of the norm (a shift in proprioceptive bias of nearly $30^{\circ}$ rightward). Informed consent was given and the experiment was performed in accordance with the ethical guidelines set by the York Human Participants Review SubCommittee, York University's Ethics Review Board and the standards of the Canadian Tri-Council Research Ethics guidelines. 
General experimental setup

Subjects were seated in a chair adjustable in both height and distance, ensuring that they could comfortably see and reach to all target positions presented on an opaque and reflective surface; a view of the setup is presented in Fig. 1a. Once the chair was adjusted, the position was fixed and remained consistent for all experimental sessions. Subjects were instructed to grasp the vertical handle of a two-joint robot manipulandum (Interactive Motion Technologies) with their right hand. Visual stimuli were projected from a monitor (SyncMaster model 510N; refresh rate: $72 \mathrm{~Hz}$; Samsung, Brisbane, CA) installed $17 \mathrm{~cm}$ above the robot and viewed by subjects as a reflected image. The position of the manipulandum's gripped-handle was recorded throughout all reaching trials at a sampling rate of $50 \mathrm{~Hz}$ and a spatial accuracy of $0.1 \mathrm{~mm}$. The room lights were dimmed and the reflective surface was positioned so that images displayed on the monitor appeared to lie in the same horizontal plane as that of the robot handle. Subjects' view of their right hand was obstructed by the reflective surface and a black cloth draped between the experimental setup and their shoulders.

\section{General procedure}

The experiment consisted of two separate testing sessions, each conducted on two consecutive days within 24 hours from one another. The first session consisted of two blocks (Fig. 2, first 2 rows), which included 3 types of tasks per block. In Block 1 subjects completed reach training trials with an 
aligned cursor, during which the visual cursor was accurately aligned with the subject's hand, followed by open-loop reaches made without visual feedback (No-Cursor task) and measures of hand proprioception (Proprioceptive Estimates task). The latter was followed immediately by Block 2, where reach training involved a cursor that was gradually rotated $45^{\circ}$ counter clockwise (CCW) relative to the hand. On the next day, subjects were re-tested on the No-Cursor reaches to measure persisting aftereffects, as well as on the Proprioceptive Estimates task to measure whether changes in felt hand position from the previous day had been retained.

\section{Reach training}

During this task, subjects made reaching movements to a yellow target circle $(1 \mathrm{~cm}$ in diameter) displayed on the reflective surface. The 3 visual targets were either set at $30^{\circ} \mathrm{CW}, 0^{\circ}$ or $30^{\circ} \mathrm{CCW}$ relative to the body's midline and located $10 \mathrm{~cm}$ radially from the home position. The order of the targets was pseudo-randomized such that subjects had reached once to each of the 3 targets before any target was repeated. The position of the subject's unseen hand was represented by a green circular cursor, also $1 \mathrm{~cm}$ in diameter, which was aligned (Block 1) or rotated (Block 2) (Fig. 1b) relative to the hand's actual location. During Block 1 subjects made 60 reaches (Fig. 2, top row, box 1) to the visual target, while in Block 2, they made 150 reaches before proprioceptive estimates and 30 reaches afterwards (Fig. 2, middle row, boxes 1 and 5). 
Trials began with the subject's hand at the home position $40 \mathrm{~cm}$ from their body midline, and after $300 \mathrm{~ms}$, a yellow target appeared. Subjects were then asked to reach towards the target while moving the robot-handle as quickly and accurately as possible, so as to align both circles. For each trial, the cursor became visible only after the handle was moved $4 \mathrm{~cm}$ away from the home position so that subjects had likely reached peak velocity prior to the onset of visual feedback.

The reach was completed once the cursor's center was positioned within $0.5 \mathrm{~cm}$ of the target's center. At that point, both circles disappeared and subjects moved their hand back to the home position via a robot-generated grooved pathway. If any attempt to move outside the established path was made, a resistance force (proportional to the depth of penetration with a stiffness of 2 $\mathrm{N} / \mathrm{mm}$ and a viscous damping of $5 \mathrm{~N} /(\mathrm{mm} / \mathrm{s}))$ was generated perpendicular to the grooved wall (Henriques and Soechting 2003).

\section{No-Cursor reaches}

The No-Cursor task was similar to the Reach Training task, except subjects were instructed to reach towards the target upon presentation without cursor feedback of their unseen hand. The task consisted of 15 trials and was administered before and after the Proprioceptive Estimates task, in each block,

on the first day (Fig. 2, first and middle row, boxes 2 and 4 in each row). In addition, No-Cursor reaches were measured once more before the proprioceptive task on Day 2 as a final measure of reach adaptation (Fig. 2, last 
row, box 1). Throughout the experiment, aftereffects were measured 5 separate times; each of these times will be numbered and referred to as epochs. After subjects' endpoint position was maintained for $500 \mathrm{~ms}$, the visual target disappeared, subjects moved their hand back to the home position via a grooved pathway, and the trial was considered complete.

\section{Proprioceptive estimates}

The Proprioceptive Estimates task was used to measure subjects' felt hand location. A trial began with the illumination of the home position indicated by a green $1 \mathrm{~cm}$ circle for $500 \mathrm{~ms}$. Subjects were instructed to wait until the green circle disappeared to push their hand outwards along a constrained, robotgenerated linear pathway described earlier (Fig. 1c). Once their hand arrived at the end of the path, one of three visual markers, represented by a yellow circle ( 1 $\mathrm{cm}$ in size) located at $30^{\circ} \mathrm{CW}, 0^{\circ}$, and $30^{\circ} \mathrm{CCW}$ relative to the body's midline, appeared. Alternatively, on one-quarter of the trials we used a proprioceptive marker, such that rather than a visual marker an auditory cue was heard at the end of the pathway, which indicated to subjects that they were to estimate their hand position with respect to their perceived body midline. Without time constraints, subjects then made a two-alternative forced-choice (2-AFC) judgment about the position of their hand (left or right) relative to the reference marker using a keyboard with their free left hand. To begin the next trial, they then moved the robot directly back to the home position along the robotgenerated linear route. Subjects completed 200 trials per task, administered once 
in each block on Day 1 (Fig. 2, top and middle row, box 3) and once on Day 2 (Fig. 2, last row, box 2). The position of the hand with respect to each reference marker was adjusted over trials using an adaptive staircase algorithm (Kesten 1958; Treutwein 1995), as described by Cressman and Henriques (Cressman and Henriques 2009).

\section{a}
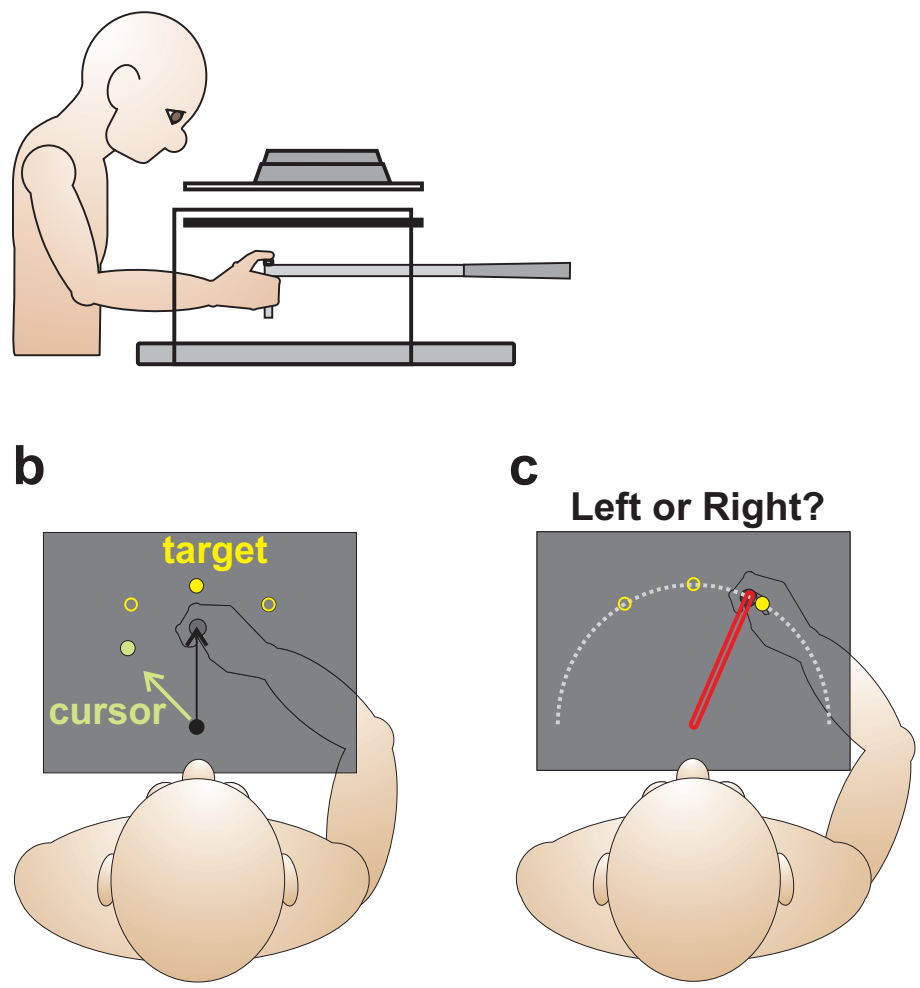

Fig. 1 Experimental setup and design. a: Side view of the experimental setup. b and c: Top view of experimental surface visible to subjects. b: Visuomotor distortion introduced in the misaligned reach training task. The $1 \mathrm{~cm}$ green cursor representing the hand was gradually rotated $45^{\circ} \mathrm{CCW}$ relative to the subject's actual hand location (black ring). Reach targets (yellow ring) $1 \mathrm{~cm}$ in size were located $10 \mathrm{~cm}$ from the home position (black circle) at $30^{\circ} \mathrm{CW}, 0^{\circ}, 30^{\circ}$ $\mathrm{CCW}$ relative to the body midline. C: In the proprioceptive estimates task, subjects actively pushed their hand out $10 \mathrm{~cm}$ along a constrained linear path (depicted in red) from the home position and judged the position of their hand with respect to a reference marker (yellow rings). Reference markers were either visual $\left(30^{\circ} \mathrm{CW}, 0^{\circ}, 30^{\circ} \mathrm{CCW}\right.$ relative to the midline), or proprioceptive, prompted by an auditory cue, wherein subjects judged the position of their hand relative to their perceived midline. 


\section{Day 1}

\section{Block 1- Aligned Cursor}

\begin{tabular}{|c|c|c|c|}
\hline \multicolumn{2}{|c|}{ Reach Training } & \multicolumn{2}{|c|}{ Proprioceptive Estimate } \\
\hline $\begin{array}{l}\text { Reach Training } \\
\text { (aligned cursor) } \\
60 \text { Trials } \\
\text { (3 target) }\end{array}$ & $\begin{array}{l}\text { No-Cursor } \\
\text { Reaches } \\
\text { (aftereffects) } \\
15 \text { Trials } \\
\text { (3 tarqet) }\end{array}$ & $\begin{array}{l}\text { Proprioceptive } \\
\text { Estimates } \\
200 \text { Trials } \\
\text { (4 marker) }\end{array}$ & $\begin{array}{c}\text { No-Cursor } \\
\text { Reaches } \\
\text { (aftereffects) } \\
15 \text { Trials } \\
\text { (3 target) }\end{array}$ \\
\hline
\end{tabular}

\section{Block 2- Rotated Cursor}

Reach Training Proprioceptive Estimate + Reach Task

\begin{tabular}{|c|c|c|c|c|}
\hline $\begin{array}{c}\text { Reach Training } \\
\text { (rotated cursor) } \\
150 \text { Trials } \\
\text { (3 target) }\end{array}$ & $\begin{array}{c}\text { No-Cursor } \\
\text { Reaches } \\
\text { (aftereffects) } \\
15 \text { Trials } \\
\text { (3 target) }\end{array}$ & $\begin{array}{l}\text { Proprioceptive } \\
\text { Estimates } \\
200 \text { Trials } \\
\text { (4 marker) }\end{array}$ & $\begin{array}{c}\text { No-Cursor } \\
\text { Reaches } \\
\text { (aftereffects) } \\
15 \text { Trials } \\
\text { (3 target) }\end{array}$ & $\begin{array}{c}\text { Reach Training } \\
\text { (rotated cursor) } \\
30 \text { Trials } \\
\text { (3 target) }\end{array}$ \\
\hline
\end{tabular}

\section{Day 2 ( 24 hrs)}

\section{Proprioceptive Estimate}

\begin{tabular}{|c|c|}
\hline No-Cursor & Proprioceptive \\
Reaches & Estimates \\
(aftereffects) & 200 Trials \\
15 Trials & (4 marker) \\
(3 target) & \\
\hline
\end{tabular}

Fig. 2 Schematic showing the order in which tasks were completed and the number of trials completed per task. Tasks run during Block 1 provided baseline measures of performance, wherein the subject's unseen hand was aligned with the cursor during reach training (first box, top row). In Block 2 the cursor was gradually rotated $45^{\circ} \mathrm{CCW}$ relative to the subject's actual hand location during reach training (first and last box, second row). On Day 2 subjects were only tested on open-loop No-Cursor reaches and proprioceptive estimates without any reach training. 


\section{Data Analyses}

\section{No-Cursor reaches}

We investigated whether subjects adapted their reaches after training with a rotated cursor and if this adaptation was maintained 24 hours later by looking at No-Cursor reaches (i.e aftereffects). Reach endpoint errors were defined as the angular deviation between a movement vector (from the home position to the reach endpoint) and a reference vector (from the home position to the target). Similarly, reach errors at peak velocity were defined as the angular difference between a movement vector at peak velocity and a reference vector. We conducted a 4 epoch (Average Aligned, Rotated Time 1, Rotated Time 2, Retention) $\times 3$ target $\left(0^{\circ}, 30^{\circ} \mathrm{CW}, 30^{\circ} \mathrm{CCW}\right)$ repeated measures analysis of variance (RM-ANOVA), and then reported our pre-planned comparisons between these levels to determine if adaptation had occurred and if this adaptation was retained. A Bonferroni correction was applied to all post-hoc pair-wise comparisons and all ANOVA results are reported with Greenhouse-Geisser corrected $p$ values to compensate for violations of sphericity.

\section{Proprioceptive estimates}

The position at which subjects perceived their hand to be aligned with the reference marker was measured by fitting their responses to a logistic function for each of the four reference marker locations. Biases, which are the measure of accuracy of a subject's proprioceptive sense of hand position, were then calculated. It is also understood as the probability of reporting left or right equally 
often $(50 \%)$. A subject's bias for a particular marker was excluded if they surpassed +2 SD (14 out of 552 marker values were replaced with their respective mean).

Proprioceptive bias was analysed comparing those measured after aligned and misaligned training on Day 1 and again on Day 2 (Day-1 Aligned, Day-1 Rotated, Day-2 Retention) and marker location $\left(30^{\circ} \mathrm{CW}, 0^{\circ}, 30^{\circ} \mathrm{CCW}\right.$, or proprioceptive midline) using a RM-ANOVA. A Bonferroni correction was applied to all post-hoc pair-wise comparisons and all ANOVA results are reported with Greenhouse-Geisser corrected $p$ values to compensate for violations of sphericity.

\section{Results}

Visuomotor adaptation

Mean baseline-subtracted open-loop reaches (aftereffects) from the NoCursor reach task are displayed in Fig. 3a. To study retention of motor adaptation after training with a misaligned cursor, we examined mean baseline aftereffects compared to aftereffects measured before and after proprioceptive estimates on Day 1 (henceforth referred to as the first and second session, respectively) and at the outset on Day 2. Analysis of mean reach endpoint errors revealed that they differed significantly between epochs $(F(2.4,108.8)=67.5, p<.001)$. Post-hoc analysis revealed a significant difference between No-Cursor reaches from the first session (Epoch 3) and baseline reaches $(p<.001)$, wherein subjects' 
reaching movements were deviated on average $11.8^{\circ}$ (approximately $26 \%$ of the distortion) more rightward, in the expected direction of motor adaptation (Fig. 3, dark grey bar). These results show that subjects adapted their reaches in response to reach training with a misaligned cursor. The magnitude of the aftereffects measured on Day 1 decreased significantly with time. When tested in a second session (Epoch 4), after subjects had performed the Proprioceptive Estimates task (Epoch 4), mean endpoint errors had been reduced to approximately $4.7^{\circ}$ (Fig. 3, light grey bar). Although smaller, these No-Cursor reaches made in the fourth epoch were still significantly deviated in the direction of adaptation, compared to baseline measures $(p<.001)$. Interestingly, rather than observing an abrupt decay from the first to the second session, there was a gradual decline in the magnitude of endpoint error across trials of two flanking epochs on the first day (Fig. 3b). During the second session, this continuous reduction plateaued at approximately $5^{\circ}$ in the direction of adaptation, relative to baseline performance, and interestingly, was still present at similar levels when re-tested the next day (Fig. 3b, Epoch 4 vs. 5). Specifically, aftereffects measured on Day 2 were relatively the same size as those in the last set of NoCursor reaches on Day 1 and thus did not significantly differ from one another (Fig. 3a, light grey vs. white bar) $(p=.05)$. Aftereffects from Day 2 relative to the baseline results were also significantly different $(p<.001)$.

In sum, these results confirm that motor adaptation on Day 1 was retained on Day 2, such that No-Cursor reaches from both sessions on Day 1 and those measured on Day 2 were significantly deviated compared to baseline reaches ( $p$ 
$<.001)$. Reach aftereffects at peak velocity displayed similar results to that of endpoint reach errors (Fig. 3a, diamonds).

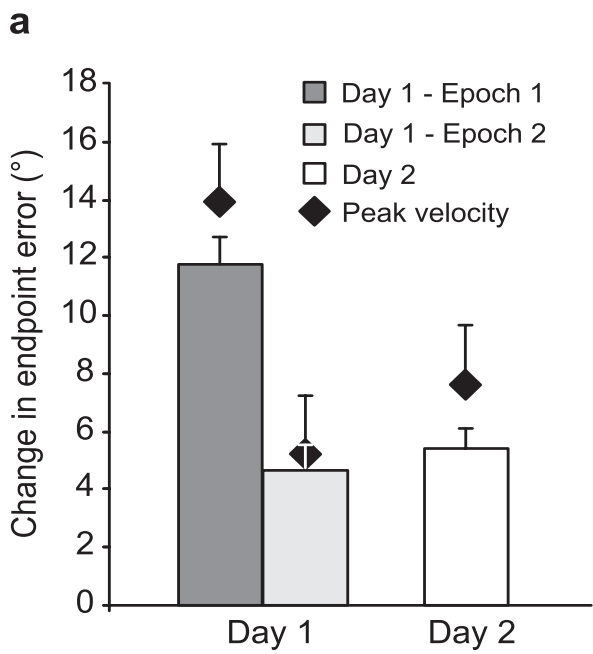

b
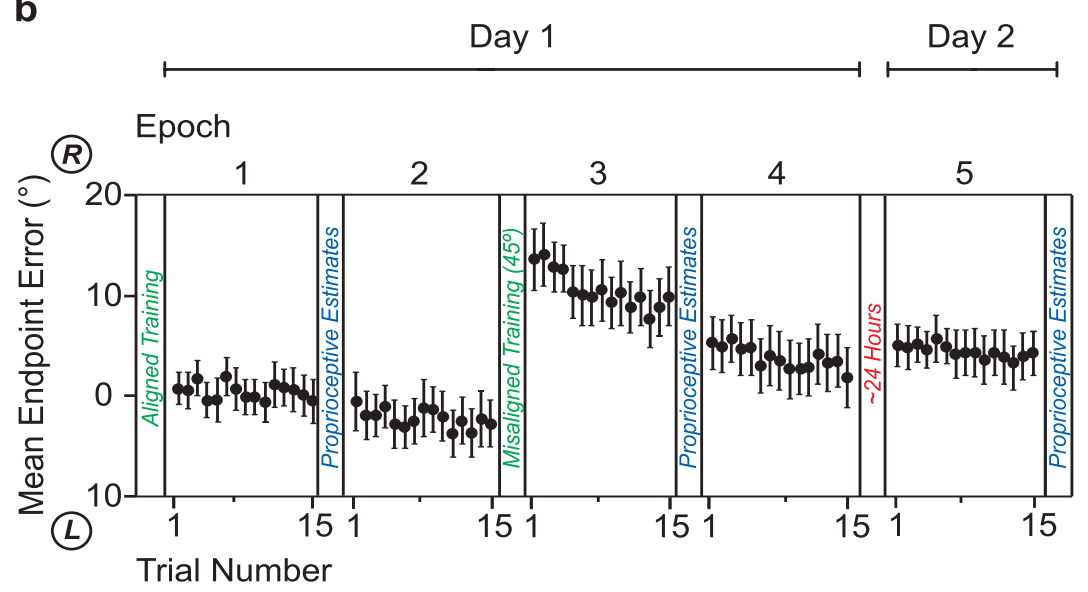

Fig. 3 a: Reach aftereffects seen as changes in endpoint error immediately after (Day 1) and 24 hours after (Day 2) training with misaligned feedback of the hand. These changes in endpoint errors were calculated by subtracting angular reach endpoint errors in the No-Cursor reach task, after training with an aligned cursor, from errors on Day 1 and 2 after training with a misaligned cursor. Dark grey bars show the change in hand movement before proprioceptive estimates (Epoch 1) and light grey bars show the change in endpoint error following proprioceptive estimates (Epoch 2). Errors at peak velocity were calculated (diamonds) by subtracting baseline measures of error at peak velocity from those obtained following training with a misaligned cursor. b: Mean endpoint error per trial in each epoch. $R$ and $L$ are used to indicate rightward and leftward directions, respectively. We see a gradual decline in adaptation, which plateaus around $5^{\circ}$ rightward, in the direction of adaptation (Epoch 3, 4), which may be due to a lack of interleaved reach training. Motor adaptation is maintained around $5^{\circ}$ when retested the following day (Epoch 5). Error bars reflect SEM. 
Proprioceptive estimates of hand position

Estimates of hand position following visuomotor adaptation were analysed to investigate proprioceptive recalibration both immediately following training and 24 hours later. Figure 4a depicts the positions at which subjects perceived their hand to be aligned with each reference marker following aligned reach training (diamonds), rotated reach training (triangles), and 24 hours later (squares). Baseline measures of felt hand position revealed a slight leftward bias, which has been seen in our previous studies and been suggested to arise due to a systematic hand bias (Jones et al. 2010; Salomonczyk et al. 2012; Salomonczyk et al. 2013). Mean bias estimates differed significantly when comparing those measured after aligned and misaligned training on Day 1 and again on Day 2 $(F(1.9,87.9)=3.7, p=.028)$. Post-hoc analysis revealed that on average, subjects showed a significant $2^{\circ}$ shift rightwards in felt hand position following rotated reach training, as compared to following aligned reach training (Fig. 4c, first white bar $),(p=.039)$. When these subjects were re-tested on the proprioceptive estimates task the following day, we observed a $2^{\circ}$ shift once again in hand bias relative to baseline measures (Fig. 4c, first grey bar), suggesting that the change in bias found immediately after training was perfectly retained 24 hours later. However, this $2^{\circ}$ shift observed immediately following reach training and 24 hours later is lower than usually observed in previous experiments involving training with a misaligned cursor (Cressman and Henriques 2009; Salomonczyk et al. 2012). Upon further inspection, we noticed a number of subjects ( $n=14, \sim 30 \%$ ) who did not show a normal change in hand 
bias following training with a rotated cursor (Fig. 4d, grey circles). Since testing for retention requires an initial change that can be retained, we performed a reanalysis on a subgroup of subjects $(n=32)$ that did show a positive change in bias of at least $0.001^{\circ}$ rightward, the expected direction of adaptation, when compared to baseline performance (Fig. $4 \mathrm{~d}$, hollow circles). Figure $4 \mathrm{~b}$ depicts the positions at which subjects in the subgroup perceived their hand to be aligned with each reference marker following aligned reach training (diamonds), rotated reach training (triangles), and 24 hours later (squares). This subgroup's mean bias estimates also differed significantly when comparing bias measured after aligned and misaligned training on Day 1 and again on Day $2(F(1.7,55.6)=13.7$, $p<.001)$. Post-hoc analysis revealed that on average, they showed a significant $4.4^{\circ}$ shift rightwards in their felt hand position following rotated reach training, as compared to following aligned reach training (Fig. 4c, second white bar), $(p<$ .001). More importantly, proprioceptive recalibration for Day 2 was approximately $2.8^{\circ}$, representing a $64 \%$ retention of the $4.4^{\circ}$ change in bias seen immediately following misaligned reach training $(p=.022)$. While this change in proprioceptive bias one day after training appears smaller than those produced immediately after reach training, this reduction did not reach significance $(p=$ .189). These results suggest that changes in felt hand position following visuomotor adaptation can be partially retained up to 24 hours later, similarly to motor adaptation. 
Proprioceptive estimates of hand position were comparable across all visual and proprioceptive (body midline) reference marker locations for all subjects $(F(3,135)=2.3, p=.075)$ and the subgroup $(F(3,93)=1.04, p=.378)$.

A one-way ANOVA revealed that our subgroup did not differ from subjects that did not show a positive bias (grey circles in Fig. 4d) in their slope (i.e. uncertainty range) fitted to the proprioceptive estimates $(F(1,132)=.457, p=$ .50). Notably, when re-analyzed, this subgroup also showed similar reach aftereffects as all of the subjects (mentioned above).

We also applied a regression procedure and observed that retained mean change in aftereffects for all subjects $(\beta=-.148, p=.439)$ as well as the subgroup $(\beta=-.094, p=0.654)$ were not a significant predictor of retained mean change in proprioceptive bias. 
a

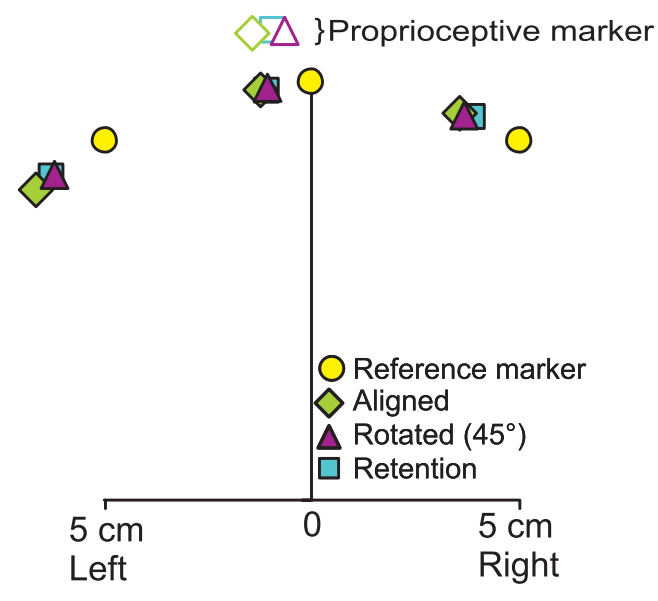

C

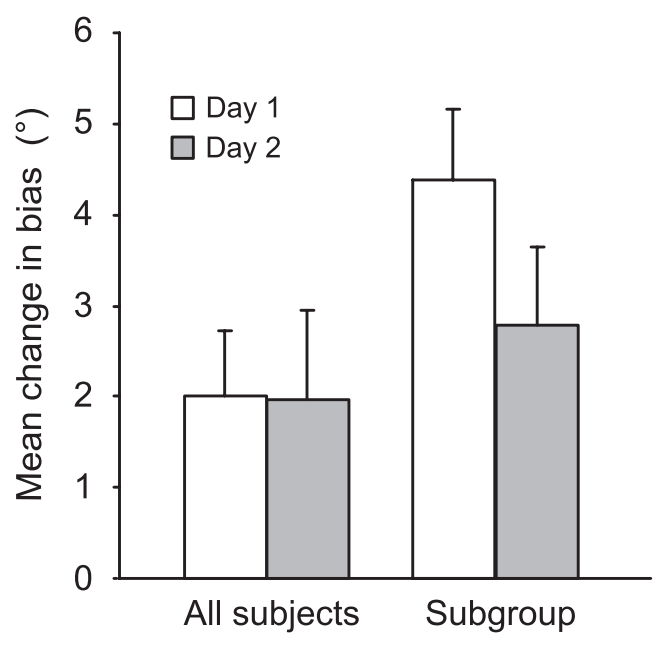

b

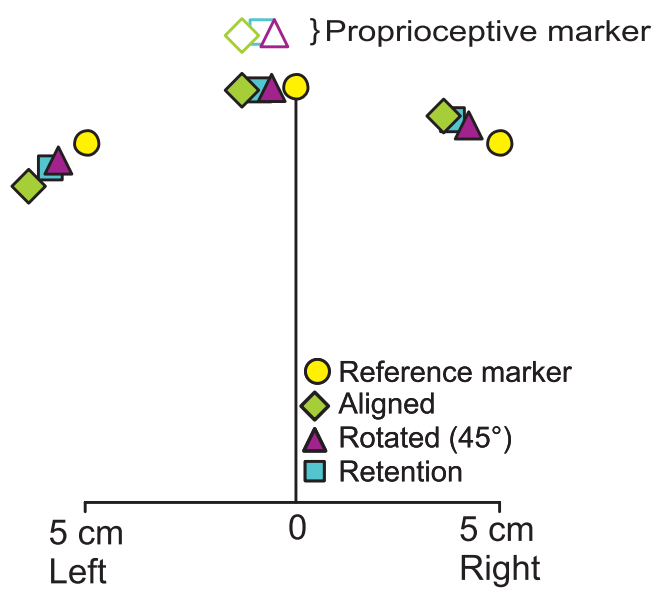

d

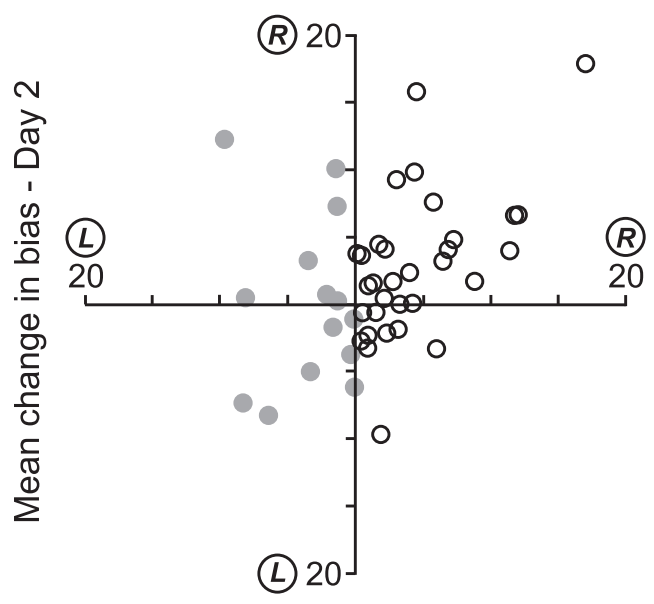

Mean change in bias - Day 1

Fig. 4 a: Mean 2-dimensional (2-D) proprioceptive biases after all subjects were exposed to aligned (diamonds) and rotated (triangle) reach training $(n=46)$. Mean change in felt hand position measured the following day is represented by squares and the circles represent actual reference marker positions (biases for the proprioceptive marker are shown as hollow shapes). b: 2-D proprioceptive biases for our subgroup $(n=32)$. c: Mean change in bias following training with a misaligned cursor (white bars) as well as 24 hours later (grey bars). The first pair of bars is the measures for all our subjects, whereas the second pair is for the subgroup. Error bars reflect SEM. d: The mean change in proprioceptive estimates, for each subject, on Day 2 is plotted as a function of the change on Day 1. $R$ and $L$ are used to indicate rightward and leftward directions, respectively. Approximately $30 \%$ of subjects did not show any change in felt hand position in the direction of adaptation (grey circles) possibly due to lack of interleaved reach training. Those who showed a change of at least $0.001^{\circ}$ in the positive direction were represented as hollow circles. 7 out of the 14 subjects who did not show a positive change on Day 1 did so 24 hours later by an average of $4.9^{\circ}$ in the direction of adaptation. 


\section{Discussion}

The goal of this study was to examine whether induced changes in subjects' felt hand position following visuomotor adaptation were robust enough to be maintained into the following day, and if so, to determine their magnitude. We assessed motor adaptation (seen as aftereffects) and proprioceptive recalibration of hand bias (seen as a change in felt hand position) immediately after training with a rotated cursor and 24 hours later. We found that, following reach training with distorted visual feedback, subjects adapted their mean openloop reaching movements by $11.8^{\circ}$, or $26 \%$ of the distortion, in the first session, compared to baseline reaches. In the second session, following proprioceptive estimates, they had deviated mean reaching trajectories by $4.7^{\circ}$, or $10.4 \%$ of the distortion, compared to baseline. When retested 24 hours later, subjects showed motor retention of these adapted hand movements from Day 1 by $5.4^{\circ}$, approximately $46 \%$ of mean aftereffects shown in the first session, and complete retention of aftereffects shown in the second session. For subjects that displayed proprioceptive recalibration in the expected direction, felt hand position shifted by $4.4^{\circ}$ compared to baseline results, and did indeed retain $64 \%$ of this change the following day. In addition, their changes in reach aftereffects were similar to those reported for the entire group. These results suggest that following visuomotor adaptation, changes affecting the sensory system, specifically proprioception, can persist beyond initial training, up to at least 24 hours. 
Proprioceptive and motor changes following visuomotor adaptation

Although both proprioceptive and motor changes occur following visuomotor adaptation (Cressman and Henriques 2009), the nature of their relationship still remains unclear. In our labs previous work, changes in subjects' felt hand position were not significantly related to changes in their aftereffects after free-reach training with a misaligned cursor (Cressman and Henriques 2009; Cressman and Henriques 2010b; Salomonczyk et al. 2011; Salomonczyk et al. 2012). However, the aforementioned relationship had not yet been investigated following an extended lapse of time. We found that proprioceptive recalibration of hand bias following visuomotor adaptation is still not significantly related to changes in aftereffects when measured approximately 24 hours after training with a misaligned cursor, suggesting that the two processes may occur concurrently through distinct processes. This idea is strongly supported in studies where specific generalization patterns occur. For instance, Mostafa and colleagues (2014), found that after training with a misaligned cursor, motor adaptation had transferred from subjects' trained right (dominant) hand to their untrained left (non-dominant) hand, however proprioceptive changes did not transfer at all between hands and was only found in the arm exposed to the distortion. Generalization patterns have also been explored by Cressman and Henriques (2010a) wherein subjects' hand movements and proprioceptive recalibration to novel targets were studied following visuomotor adaptation. They found that subjects' reaches confirmed previous studies' observations of localized and narrow generalization patterns in motor adaptation (Krakauer et al. 
2000; Wang and Sainburg 2005). Interestingly, proprioceptive recalibration generalized quite broadly in comparison, such that the magnitude of recalibration observed for the trained target direction was found at similar levels up to $90^{\circ}$ $\mathrm{CCW}$ from the trained direction. They also found that after training with small cursor-gains, subjects' reaches had been adapted, whereas their felt hand position was not recalibrated. They suggest that these generalization patterns in sensory and motor plasticity may be the result of two respective error signals processed in different areas of the brain: one that arises from a discrepancy between desired and actual movement, mainly responsible for motor changes, and one from a discrepancy between visual and proprioceptive estimates of hand position, mainly responsible for sensory changes.

\section{Retention of motor adaptation}

Consistent with previous studies, subjects' reaches following visuomotor adaptation remained deviated in the direction of adaptation up to one day later. Although most studies measure retention through facilitation, in the form of savings or increased re-adaptation rates, we wished to compare changes in sensory estimates with those changes observed in motor endpoint error. Mean aftereffects observed only 24 hours later compared to the mean of those immediately following rotated reach training were smaller, with only $46 \%$ retained, in comparison to those observed up to an entire year later as found by Yamamoto et al. (2006), who showed up to $91 \%$ retention relative to the initial post-test. One explanation for the latter's high to near perfect retention of 
aftereffects after an extended period of time may have been due to the joystick paradigm itself, whereas similar results may be less likely for reaches which are less restrained to a particular tool. Specifically, certain perturbations may provide a sufficiently distinct context such that everyday movements would not interfere with learning and retention following adaptation (Brashers-Krug et al. 1996; Wolpert et al. 1998; Krakauer et al. 1999).

Aftereffects were measured following training with a rotated cursor both before (Epoch 3) and right after (Epoch 4) the Proprioceptive Estimates task, which took approximately $25-30$ minutes to complete. The smooth continuous decrease in reach adaptation across trials suggests that this estimation task between the two epochs did not cause nor accelerate this decay, and that the smaller aftereffects seen in Epoch 4 are likely due to a gradual washout. In some of our other studies on this topic, we try to maintain the same level of reach adaptation by interleaving rotated-cursor training with proprioceptive estimate trials; a technique that others have also used for similar purposes (Kesten 1958; Simani et al. 2007; Synofzik et al. 2008; Ostry et al. 2010). Nonetheless, this continuous decrease in reach aftereffects appears to saturate at approximately $5^{\circ}$ on Day 1 (Epoch 4) and seems to be maintained into the following day at a similar level (Fig. 3b, Epoch 5). Specifically, when measured 24 hours later, aftereffects from the second epoch (Epoch 4) after training on Day 1 were almost completely preserved on Day 2. Smith et al. (2006) describes two adaptive processes that result from learning: a fast-learning and a slow-learning process. Since the fast-learning process is thought to decay much earlier than its 
counterpart, its gradual waning would reveal the slow-learning process that is thought to be responsible for savings and anterograde interference (Shadmehr et al. 2010). It is possible that aftereffects observed immediately after training, in the first session (Epoch 3), may account for a combination of the two processes including this fast-learning process of adaptation highly involved in initial learning, whereas those observed after the gradual decay are the resulting aftereffects from slow-learning process. Furthermore, it is these robust aftereffects resulting form the slow-learning process, accounting for $40 \%$ of the first set of aftereffects, which are retained the next day. In a study by Joiner and Smith (2008), a forcefield adaptation task was used to study the relationship between initial learning and long-term retention; retention measured 24 hours later was observed to have a very similar shape as the model-predicted slow learning process $(r=0.99)$, suggesting that the slow process contributes strongly to retention of motor memories, whereas the fast-process does not.

Our results can also be explained by Berniker and Kording's (2008) source-estimation model, based on how a person's nervous system attributes observed movement errors, such as when adapting to a perturbation. Specifically, does one principally assign the source of errors to changes in worldbased or body-based properties? Depending on the source of the motor errors, motor adaptation can vary from a transient to a more long-term change. The rather quick decrease in initial aftereffects seen immediately following visuomotor adaptation (Fig. 3b, Epoch 3) may principally reflect world-based updates as the subject adapts their movements. The retention of motor changes such as those 
seen on the second day in Epoch 5 (Fig. 3b), as well as the last set of aftereffects (Epoch 4), may strongly reflect an update of the visuomotor mapping that is more specific to the body than world-based, and thus is a more persistent form of adaptation. Additionally, given our findings in this study on the persistence of proprioceptive recalibration, changes in proprioceptive hand position, both immediately and the 24 hours later, may also reflect predominantly body-based source errors.

\section{Retention of proprioceptive recalibration}

Our main interest for this study was the degree of retention with regard to changes in proprioceptive hand position following visuomotor adaptation, which had not been investigated to date. When we analysed our entire group of subjects, we found that, on average, individuals significantly recalibrated their sense of hand position to be $2^{\circ}$ more to the right (the direction of motor adaptation) compared to baseline measures. When tested the following day, subjects showed perfect retention $(99.6 \%$ retained) of this shift in felt hand position. However, given the smaller magnitude following adaptation compared to our previous papers $\left(\sim 6^{\circ}\right.$ change with a $30^{\circ}$ distortion) (Cressman and Henriques 2009; Cressman and Henriques 2010b; Cressman et al. 2010; Salomonczyk et al. 2011), we investigated further and noticed a group of subjects who did not show a shift in the expected direction following training with a visuomotor discrepancy (Fig. 4d, grey circles), despite having equivalent slopes (or uncertainty ranges) as the others, confirming correct performance of the task. 
These subjects may not have shown expected recalibration due to a lack of interleaved reach training, which would have helped maintain proprioceptive recalibration throughout the experiment. For instance, in our previous studies that had weaved reach training trials throughout proprioceptive estimates, approximately 83 to $100 \%$ of subjects showed the expected shift in sense of hand position (Cressman and Henriques 2009; Cressman and Henriques 2010b; Cressman et al. 2010; Salomonczyk et al. 2011; Salomonczyk et al. 2012; Clayton et al. 2013), whereas in those that had not (Salomonczyk et al. 2013; Mostafa et al. 2014), only 72 to $91 \%$ of subjects showed this recalibration. However, interleaving rotated reach training with the proprioceptive estimates task in this study would have proven to be problematic, since any type of retraining on Day 2 had to be avoided in order to ensure we were testing for retention of motor and proprioceptive changes.

So that we may draw clear conclusions on the persistence of proprioceptive recalibration, we then only looked at participants (32 in total, or the remaining $70 \%$ ) who showed at least a $0.001^{\circ}$ proprioceptive shift in bias in the direction of the distortion. This allowed us to see whether participants who showed at least some changes in felt hand position continued to do so the following day. On average, the shift in the felt hand position for these 32 subjects increased to approximately $4.4^{\circ}$ and their estimates of hand position were significantly shifted by $2.8^{\circ}$, compared to baseline, 24 hours later. These results are similar to those found by Ostry et al. (2010) where subjects retained a shift of $2 \mathrm{~mm}$ in perceived limb position following 24 hours after adaptation to a force- 
field. While the size of the retention of proprioceptive recalibration in this study appears rather small, it is similar, if not larger, than the changes in felt handmotion found by Ostry et al, such that the somatosensory changes they observed were about $10 \%$ of the size of initial reach aftereffects on Day $1(\sim 20 \mathrm{~mm})$ compared to $17 \%$ for all our subjects and $24 \%$ for our subgroup.

\section{Offline gains}

Consolidation refers to the stabilization of learned memories postacquisition and can prevent decay or interference. This is done by modifying intracellular cascades at the synaptic level, synthesizing specific neuronal proteins, and reorganizing the neural networks that represent the memory (Dudai 2004). Some studies have shown that consolidation improves performance, following training, without any additional practice (termed "offline gains") and can be influenced by sleep (Robertson et al. 2005; Walker et al. 2002). In addition, memory consolidation has been shown to help reduce learned motor changes' susceptibility to disruption or interference by a second competing motor memory (Brashers-Krug and Shadmehr 1996; 1997). For instance, Walker et al. (2003) tested two different groups, one that had learned a first sequence of fingertapping movements and one that learned a second one right after the first. The first group displayed sleep-dependent enhancements such as increased speed and accuracy when tested 24 hours later. However, for the group that had learned a second sequence, interference was seen such that improvements in 
accuracy were only seen for the second tapping task they had learned and not the first. Both groups showed improvements related to speed.

Interestingly, in the current study, 7 out of the 14 subjects who did not show a change in hand position in the expected direction of adaptation immediately following visuomotor adaptation, did so 24 hours later by an average of $4.9^{\circ}$ rightwards (Fig. $4 \mathrm{~d}$, top left quadrant). It may be possible that for a small number of people, this change in proprioception benefits by offline gains.

\section{Conclusion}

In summary, subjects expectedly showed motor adaptation and proprioceptive recalibration following visuomotor adaptation. As shown in previous studies, their adapted reach movements were retained up to the next day following training with a perturbed cursor. More importantly, subjects' induced sensory changes, specifically proprioception, persisted up to 24 hours later as well. These results suggest that sensory memory may play a significant role alongside motor memory with regards to the long-term changes an individual may experience following visuomotor adaptation. Further investigation may provide insight into the relationship between both of these processes. 


\section{References}

Abeele S, Bock O (2001) Sensorimotor adaptation to rotated visual input: different mechanisms for small versus large rotations. Exp Brain Res, 140: 407-410

Admiraal MA, Keijsers NL, Gielen CC (2003) Interaction between gaze and pointing toward remembered visual targets. J Neurophysiol, 90: 21362148

Bernier PM, Chua R, Franks IM (2005) Is proprioception calibrated during visually guided movements? Exp Brain Res 167: 292-296

Berniker M, Kording K (2008) Estimating the sources of motor errors for adaptation and generalization. Nat Neurosci 11: 1454-1461

Bock O (1990) Dynamic properties of human goal-directed arm movements. Behav Brain Res 39: 240-248

Bock O, Schneider S, Bloomberg J (2001) Conditions for interference versus facilitation during sequential sensorimotor adaptation. Exp Brain Res 138: 359-365

Brashers-Krug T, Shadmehr R, Bizzi E (1996) Consolidation in human motor memory. Nature 382: 252-255

Buch ER, Young S, Contreras-Vidal JL (2003) Visuomotor adaptation in normal aging. Learning \& Memory 10: 55-63

Buneo, CA, Andersen, RA (2006) The posterior parietal cortex: Sensorimotor interface for the planning and online control of visually-guided movements. Neuropsychologia 44: 2594-2606

Burgess PR, Clark FJ (1969) Characteristics of knee joint receptors in the cat. J Physiol 203: 317-335

Caithness G, Osu R, Bays P, Chase H, Klassen J, Kawato M, Wolpert DM, Flanagan JR (2004) Failure to consolidate the consolidation theory of learning for sensorimotor adaptation tasks. J Neurosci 24: 8662-8671

Choe CS, Welch RB (1974) Variables affecting the intermanual transfer and decay of prism adaptation. J Exp Psychol 102: 1076-84

Clayton HA, Cressman EK, Henriques DY (2013) Proprioceptive sensitivity in Ehlers-Danlos syndrome patients. Exp Brain Res 230: 311-321 
Cressman EK, Henriques DY (2009) Sensory recalibration of hand position following visuomotor adaptation. J Neurophysiol 102: 3505-3518

Cressman EK, Henriques DY (2010a) Generalization patterns for motor and sensory plasticity differ following visuomotor learning. In: Society for Neuroscience San Diego, California

Cressman EK, Henriques DY (2010b) Reach adaptation and proprioceptive recalibration following exposure to misaligned sensory input. J Neurophysiol 103: 1888-1895

Cressman EK, Salomonczyk D, Henriques DY (2010) Visuomotor adaptation and proprioceptive recalibration in older adults. Exp Brain Res 205: 533-544

Cunningham HA (1989) Aiming error under transformed spatial mappings suggests a structure for visual-motor maps. J Exp Psychol Hum Percept Perform 15: 493-506

Dionne JK, Henriques DY (2008) Interpreting ambiguous visual information in motor learning. Journal of vision 8: 2 1-10

Dudai $Y$ (2004) The neurobiology of consolidations, or, how stable is the engram? Annu. Rev. Psychol 55: 51-86

Elliott D, Pollock BJ, Lyons J, Chua R (1995) Intermittent vision and discrete manual aiming. Perceptual and motor skills $80: 1203-1213$

Gonshor A, Jones GM (1976) Extreme vestibulo-ocular adaptation induced by prolonged optical reversal of vision. The Journal of physiology 256: 381414

Goodwin GM, McCloskey DI, Matthews PBC (1972) The contribution of muscle afferents to keslesthesia shown by vibration induced illusions of movements and by the effects of paralysing joint afferents. Brain 95: 705748

Harris CS (1963) Adaptation to displaced vision: visual, motor, or proprioceptive change? Science 140: 812-813

Harris CS (1965) Perceptual adaptation to inverted, reversed, and displaced vision. Psychol Rev 72: 419-444

Hay JC, Pick Jr HL (1966) Gaze-contingent prism adaptation: optical and motor factors. Journal of experimental psychology 72: 640. 
Hay JC, Pick HLJ, Ikeda K (1965) Visual capture produced by prism spectacles. Psychonomic Science 2: 215-216

Henriques DY, Soechting JF (2003) Bias and sensitivity in the haptic perception of geometry. Exp Brain Res 150: 95-108

Jeannerod M (1988) The neural and behavioural organization of goal-directed movements. Oxford University Press, USA

Joiner WM, Smith MA (2008) Long-term retention explained by a model of shortterm learning in the adaptive control of reaching. J Neurophysiol 100: 2948-2955

Jones SA, Cressman EK, Henriques DY (2010) Proprioceptive localization of the left and right hands. Exp Brain Res 204: 373-383

Kagerer FA, Contreras-Vidal JL, Stelmach GE (1997) Adaptation to gradual as compared with sudden visuo-motor distortions. Experimental brain research 115: 557-561

Kawato M (1999) Internal models for motor control and trajectory planning. Current opinion in neurobiology 9: 718-727

Kesten H (1958) Accelerated stochastic approximation. The Annals of Mathematical Statistics 29: 41-59

Klassen J, Tong C, Flanagan JR (2005) Learning and recall of incremental kinematic and dynamic sensorimotor transformations. Exp Brain Res 164: 250-259

Krakauer JW (2006) Motor learning: its relevance to stroke recovery and neurorehabilitation. Curr Opin Neurol 19: 84-90

Krakauer JW, Ghez C, Ghilardi MF (2005) Adaptation to visuomotor transformations: consolidation, interference, and forgetting. J Neurosci 25: 473-478

Krakauer JW, Ghilardi MF, Ghez C (1999) Independent learning of internal models for kinematic and dynamic control of reaching. Nat Neurosci 2: 1026-1031

Krakauer JW, Pine ZM, Ghilardi MF, Ghez C (2000) Learning of visuomotor transformations for vectorial planning of reaching trajectories. J Neurosci 20: 8916-8924 
Martin TA, Keating JG, Goodkin HP, Bastian AJ, Thach WT (1996) Throwing while looking through prisms. II. Specificity and storage of multiple gazethrow calibrations. Brain 119 ( Pt 4): 1199-1211

Mazzoni P, Krakauer JW (2006) An implicit plan overrides an explicit strategy during visuomotor adaptation. The Journal of neuroscience 26: 3642-3645

Mostafa AA, Salomonczyk D, Cressman EK, Henriques DY (2014) Intermanual transfer and proprioceptive recalibration following training with translated visual feedback of the hand. Exp Brain Res

Ostry DJ, Darainy M, Mattar AA, Wong J, Gribble PL (2010) Somatosensory plasticity and motor learning. J Neurosci 30: 5384-5393

Prablanc C, Echallier JF, Komilis E, Jeannerod M (1979) Optimal response of eye and hand motor systems in pointing at a visual target. I. Spatiotemporal characteristics of eye and hand movements and their relationships when varying the amount of visual information. Biol Cybern 35: $113-124$

Proteau L (1995) Sensory integration in the learning of an aiming task. Canadian Journal of Experimental Psychology/Revue canadienne de psychologie experimentale 49: 113

Redding GM, Rossetti Y, Wallace B (2005) Applications of prism adaptation: a tutorial in theory and method. Neurosci Biobehav Rev 29: 431-444

Redding GM, and Wallace B (1978) Sources of "overadditivity" in prism adaptation. Percept Psychophys 24: 58-62

Redding GM, and Wallace B (1988) Adaptive mechanisms in perceptual-motor coordination: components of prism adaptation. J Mot Behav 20: 242-254

Redding GM, and Wallace B (1996) Adaptive spatial alignment and strategic perceptual-motor control. J Exp Psychol Hum Percept Perform 22: 379394

Redding GM, and Wallace B (1997) Prism Adaptation During Target Pointing From Visible and Nonvisible Starting Locations. J Mot Behav 29: 119-130,

Redding GM, and Wallace B (2001) Calibration and alignment are separable: evidence from prism adaptation. J Mot Behav 33: 401-412

Redding GM, and Wallace B (2002) Strategic calibration and spatial alignment: a model from prism adaptation. J Mot Behav 34: 126-138 
Redding GM, and Wallace B (2003) Dual prism adaptation: calibration or alignment? J Mot Behav 35: 399-408

Redding GM, Wallace B (2004) First-trial adaptation to prism exposure: artifact of visual capture. J Mot Behav 36: 291-304

Redding GM, and Wallace B (2006)Generalization of prism adaptation. J Exp Psychol Hum Percept Perform 32: 1006-1022

Robertson, EM, Press, DZ, Pascual-Leone, A (2005) Off-line learning and the primary motor cortex. J. Neurosci. 25: 6372-6378

Rothwell JC, Traub MM, Day BL, Obeso JA, Thomas PK, Marsden CD (1982) Manual motor performance in a deafferented man. Brain 105: 515-542

Sainburg RL, Wang J (2002) Interlimb transfer of visuomotor rotations: independence of direction and final position information. Experimental Brain Research 145: 437-447.

Salomonczyk D, Cressman EK, Henriques DY (2011) Proprioceptive recalibration following prolonged training and increasing distortions in visuomotor adaptation. Neuropsychologia 49: 3053-3062

Salomonczyk D, Cressman EK, Henriques DY (2013) The role of the crosssensory error signal in visuomotor adaptation. Exp Brain Res 228: 313325

Salomonczyk D, Henriques DY, Cressman EK (2012) Proprioceptive recalibration in the right and left hands following abrupt visuomotor adaptation. Exp Brain Res 217: 187-196

Shadmehr R, ZM Moussavi (2000) Spatial generalization from learning dynamics of reaching movements. J Neurosci 20: 7807-15

Shadmehr R, FA Mussa-Ivaldi (1994) Adaptive representation of dynamics during learning of a motor task. J Neurosci 14(5 Pt 2): 3208-24

Shadmehr R, Brashers-Krug T (1997) Functional stages in the formation of human long-term motor memory. J Neurosci 17: 409-419

Shadmehr R, Smith MA, Krakauer JW (2010) Error correction, sensory prediction, and adaptation in motor control. Annu Rev Neurosci 33: 89-108

Simani MC, McGuire LM, Sabes PN (2007) Visual-shift adaptation is composed of separable sensory and task-dependent effects. J Neurophysiol 98: 2827-2841 
Smith MA, Ghazizadeh A, Shadmehr R (2006) Interacting adaptive processes with different timescales underlie short-term motor learning. PLoS Biol 4: e179

Sober SJ, Sabes PN (2003) Multisensory integration during motor planning. The Journal of Neuroscience 23: 6982-6992

Sober, SJ, Sabes PN (2005) Flexible strategies for sensory integration during motor planning. Nat Neurosci 8: 490-7

Taub E, Goldberg LA (1973) Prism adaptation: control of intermanual transfer by distribution of practice. Science 180: 755-7

Tong C, Wolpert DM, Flanagan JR (2002) Kinematics and dynamics are not represented independently in motor working memory: evidence from an interference study. J Neurosci 22: 1108-1113

Treutwein B (1995) Adaptive psychophysical procedures. Vision Research 35: 2503-2522

van Beers RJ, Sittig AC, van der Gon JJD (1996) How humans combine simultaneous proprioceptive and visual position information. Experimental Brain Research 111: 253-261

Van Beers RJ, Sittig AC, van der Gon JJD (1998) The precision of proprioceptive position sense. Exp Brain Res 122: 367-377

van Beers RJ, Sittig AC, van der Gon JJD (1999) Integration of proprioceptive and visual position-information: An experimentally supported model. Journal of Neurophysiology 81: 1355-1364

Walker MP, Brakefield T, Morgan A, Hobson JA, Stickgold R (2002) Practice with sleep makes perfect: sleep-dependent motor skill learning. Neuron 35: 205-211

Walker MP, Brakefield T, Hobson JA, Stickgold R (2003) Dissociable stages of human memory consolidation and reconsolidation. Nature 425: 616-620

Wang J, Sainburg RL (2005) Adaptation to visuomotor rotations remaps movement vectors, not final positions. J Neurosci 25: 4024-4030

Wise SP, Shadmehr R (2002) In: Encyclopedia of the Human Brain, Ramachandran, V. S. (ed), Academic Press: San Diego, CA, vol. 3, pp. $137-157$ 
Wolpert DM, Ghahramani Z, Jordan MI (1995) An internal model for sensorimotor integration. Science 269: 1880-1882

Wolpert DM, Miall RC, Kawato M (1998) Internal models in the cerebellum. Trends Cogn Sci 2: 338-347

Woodsworth, RS (1899) The accuracy of voluntary movement. Psyc Rev: Monograph Supplements 3: i-114

Yamamoto K, Hoffman DS, Strick PL (2006) Rapid and long-lasting plasticity of input-output mapping. J Neurophysiol 96: 2797-2801

Yin PB, Kitazawa S (2001) Long-lasting aftereffects of prism adaptation in the monkey. Exp Brain Res 141: 250-253 


\title{
Appendix A: Subject form
}

\author{
Informed Consent Form (for unpaid participants) \\ Date: Study Name: Multisensory interaction in motor control and learning \\ Researchers: Nilufer Nourouzpour (MSc) ${ }^{1,2}$, Danielle Salomonczyk(PhD) ${ }^{2,3}$, Erin K. Cressman $(\mathrm{PhD}$; Co- \\ Supervisor) ${ }^{4}$, and Denise Y. P. Henriques (Principal Investigator) ${ }^{1,2,3}$ \\ ${ }^{1}$ School of Kinesiology and Health Science, York University, Toronto, Canada \\ ${ }^{2}$ Centre for Vision Research, York University, Toronto, Canada \\ ${ }^{3}$ Department of Psychology, York University, Toronto, Canada \\ ${ }^{4}$ School of Human Kinetics, University of Ottawa, Ottawa, Canada
}

What You Will Be Asked to Do in the Research: You will be asked to reach or point toward visual targets displayed on a screen and/or point to your unseen other hand (felt target). In most tasks, you will be sitting comfortable in a chair, but some tasks, you will sit in a chair that swivels left and right while you aim your hand to a target.

Risks and Discomforts: We do not foresee any risks or discomfort from your participation in the research. Benefits of the Research and Benefits to You: You will receive 3 credits for participation in this study.

Voluntary Participation: Your participation in the study is completely voluntary and you may choose to stop participating at any time. Your decision not to volunteer will not influence your relationship with us or anyone else at York University either now, or in the future.

Withdrawal from the Study: You can stop participating in the study at any time, for any reason, if you so decide. If you decide to stop participating, you will still be eligible to receive the promised pay for agreeing to be in the project. Your decision to stop participating, or to refuse to answer particular questions, will not affect your relationship with the researchers, York University, or any other group associated with this project. In the event you withdraw from the study, all associated data collected will be immediately removed from our computers.

Confidentiality: All information you supply and recording of your arm movements or judgments about hand location during the experiment will be held in confidence, your name will not appear in any report or publication of the research. Your data will be safely stored password protected computers in our locked laboratory and only research staff will have access to this information. We will keep your information and recording will be destroyed after the study has been published. Confidentiality will be provided to the fullest extent possible by law.

Questions About the Research? If you have questions about the research in general or about your role in the study, please feel free to contact Dr. Denise Henriques either by telephone at (416) 736-2100, extension 77215 or by e-mail (deniseh@yorku.ca). This research has been reviewed and approved by the Human Participants Review Sub-Committee, York University's Ethics Review Board and conforms to the standards of the Canadian Tri-Council Research Ethics guidelines. If you have any questions about this process, or about your rights as a participant in the study, please contact the Sr. Manager \& Policy Advisor for the Office of Research Ethics, $5^{\text {th }}$ Floor, York Research Tower, York University (telephone 416-736-5914 or e-mail ore@yorku.ca). If you have any questions the Graduate Program in Kinesiology and Health Science office may also be contacted at 341 Bethune College, York University (telephone 416-736-5728 or e-mail kahs@yorku.ca).

\section{Legal Rights and Signatures:}

I__ consent to participate in this study conducted by Dr. Denise Henriques and her research team. I have understood the nature of this project and wish to participate. I am not waiving any of my legal rights by signing this form. My signature below indicates my consent.

Signature

Participant
Date

Date 